\title{
NÖRLUND METHODS OF SUMMABILITY ASSOCIATED WITH POLYNOMIALS
}

\author{
by D. BORWEIN \\ (Received 8th September 1959)
}

\section{Introduction}

Let $s, s_{n}(n=0,1, \ldots)$ be arbitrary complex numbers, and let

$$
p(z)=p_{0}+p_{1} z+\ldots+p_{j} z^{j}
$$

be a polynomial, with complex coefficients, which satisfies the normalizing condition

$$
p(1)=1 \text {. }
$$

Associated with such a polynomial is a Nörlund method of summability $N_{p}$ : the sequence $\left\{s_{n}\right\}$ is said to be $N_{p}$-convergent to $s$, and we write $s_{n} \rightarrow s\left(N_{p}\right)$, if

$$
\lim _{n \rightarrow \infty} \sum_{v=0}^{j} p_{v} s_{n-v}=s .
$$

Evidently the method is regular, i.e. $s_{n} \rightarrow s\left(N_{p}\right)$ whenever $s_{n} \rightarrow s$.

Let $q(z)=q_{0}+q_{1} z+\ldots+q_{k} z^{k}, q(1)=1$.

For convenience, we suppose throughout that $p_{n}=0$ for $n>j$ and $q_{n}=0$ for $n>k$, so that

and

$$
\begin{gathered}
p(z)=\sum_{n=0}^{\infty} p_{n} z^{n}, \quad q(z)=\sum_{n=0}^{\infty} q_{n} z^{n}, \\
\sum_{\nu=0}^{n} p_{v} s_{n-v}=\sum_{v=0}^{j} p_{v} s_{n-v} \text { for } n>j,
\end{gathered}
$$

$$
\sum_{v=0}^{n} q_{v} s_{n-v}=\sum_{\nu=0}^{k} q_{v} s_{n-v} \text { for } n>k .
$$

The object of this note is to investigate some of the properties of Nörlund methods associated with polynomials. We shall also be concerned with the Cesàro method $(C, \alpha)$, the Abel method $A$, and the "product" methods $(C, \alpha) N_{p}$ and $A N_{p}$; the latter two methods being defined as follows. The sequence $\left\{s_{n}\right\}$ is $(C, \alpha) N_{p}$-convergent to $s$ if $t_{n}=\sum_{\nu=0}^{n} p_{\nu} s_{n-v} \rightarrow s(C, \alpha)$; it is $A N_{p}$-convergent to $s$ if $t_{n} \rightarrow s(A)$.

A summability method $X$ is said to include a method $Y$ if the $Y$-convergence of any sequence to $s$ implies its $X$-convergence to $s$. The methods are said to be equivalent if each includes the other.

Throughout the note it should be borne in mind that the Nörlund methods $N_{p}$ and $N_{q}$, being associated with the polynomials $p(z)$ and $q(z)$, are not of the most general type (see (2), § 4.1). 


\section{Simple Theorems Concerning Inclusion}

We defer the statement of the main theorems till $\S 3$ and proceed to prove some simpler results.

Theorem 1. There is a sequence which is $(C, \alpha)$-convergent for every $\alpha>0$ but not $N_{p}$-convergent.

Proof. If $|z|=1, z \neq 1, p(1 / z) \neq 0$, then, for $n>j$,

$$
\sum_{v=0}^{n} p_{v} z^{n-v}=z^{n} p(1 / z)
$$

which oscillates as $n$ tends to infinity; and so the sequence $\left\{z^{n}\right\}$ is not $N_{p^{-}}$ convergent, but as is well known, it is $(C, \alpha)$-convergent to 0 for every $\alpha>0$.

Corollary. $N_{p}$ does not include $(C, \alpha)$ for any $\alpha>0$.

Theorem 2. The method $N_{f}$, associated with the polynomial $f(z)=p(z) q(z)$, includes both $N_{p}$ and $N_{q}$.

Proof. (Cf. the proof of Theorem 17 in (2)). Let $t_{n}=\sum_{v=0}^{n} p_{v} s_{n-v}$, and note that $f(z)=\sum_{n=0}^{\infty} f_{n} z^{n}$ where $f_{n}=\sum_{v=0}^{n} p_{v} q_{n-v}$. Then

$$
\sum_{\nu=0}^{n} f_{v} s_{n-v}=\sum_{\nu=0}^{n} q_{v} t_{n-v}
$$

which tends to $s$ whenever $t_{n} \rightarrow s$, i.e. $N_{f}$ includes $N_{p}$. Similarly, $N_{f}$ includes $N_{q}$.

Corollary. The methods $N_{p}$ and $N_{q}$ are consistent, i.e. if $s_{n} \rightarrow s\left(N_{p}\right)$ and $s_{n} \rightarrow s^{\prime}\left(N_{q}\right)$, then $s=s^{\prime}$.

From Theorem 2 we can at once deduce a result of Silverman and Szasz ((4), Theorem 14), namely that, if $p(z)=\left(1+z+\ldots+z^{j}\right) /(1+j), q(z)=$ $\left(1+z+\ldots+z^{k}\right) /(1+k)$, then a sufficient condition for $N_{q}$ to include $N_{p}$ is that $1+j$ should be a factor of $1+k$. Theorem I (below) shows that the condition is also necessary. The next theorem is a generalisation of another of their results ((4), Theorem 15).

Theorem 3. If $h(z)$ is the highest common factor of $p(z)$ and $q(z)$, normalized so as to make $h(1)=1$, then a necessary and sufficient condition for a sequence to be both $N_{p}$ - and $N_{q}$-convergent is that it be $N_{h}$-convergent.

Proof. That the condition is sufficient follows from Theorem 2. To prove that it is necessary, we observe that there are polynomials

$$
a(z)=\sum_{n=0}^{\infty} a_{n} z^{n}, \quad b(z)=\sum_{n=0}^{\infty} b_{n} z^{n}
$$

such that

$$
h(z)=a(z) p(z)+b(z) q(z)=\sum_{n=0}^{\infty} h_{n} z^{n}
$$


say. Hence if $t_{n}=\sum_{v=0}^{n} p_{v} s_{n-v} \rightarrow s$ and $u_{n}=\sum_{v=0}^{n} q_{v} s_{n-v} \rightarrow s$, then

$$
\sum_{v=0}^{n} h_{v} s_{n-v}=\sum_{\nu=0}^{n} a_{v} t_{n-v}+\sum_{\nu=0}^{n} b_{v} u_{n-v} \rightarrow s a(1)+s b(1)=s,
$$

since $h(1)=p(1)=q(1)=1$. The required result follows.

\section{The Main Theorems}

It is to be supposed throughout the rest of the note that

$$
p(0) \neq 0 .
$$

This restriction is not a serious one, since, if $r$ is a positive integer and $f(z)=z^{r} p(z)=\sum_{v=0}^{j+r} f_{v} z^{v}$, then $\sum_{v=0}^{n+r} f_{v} s_{n+r-v}=\sum_{\nu=0}^{n} p_{v} s_{n-v}$ so that $N_{p}$ and $N_{S}$ are equivalent.

Theorem I. In order that $N_{q}$ should include $N_{p}$ it is necessary and sufficient that $q(z) / p(z)$ should not have poles on or within the unit circle.

Theorem II. If $q(z) / p(z)$ has poles of maximum order $m$ on the unit circle and does not have poles within the unit circle, then $(C, m) N_{q}$ includes $N_{p}$, but, for any $\varepsilon>0$, there is an $N_{p^{-}}$-convergent sequence which is not $(C, m-\varepsilon) N_{q^{-}}$ convergent.

Theorem III. If $q(z) / p(z)$ has a pole within the unit circle, then there is an $N_{p}$-convergent sequence which is not $A N_{q}$-convergent.

Noting that $(C, 0)$ is identical with $N_{q}$ when $q(z)$ is 1 (i.e. $q_{0}=1, q_{n}=0$ for $n>0$ ), and that $N_{p}$ always includes $(C, 0)$, we obtain the following corollaries of the theorems.

$\mathbf{I}^{\prime}$. In order that $N_{p}$ should be equivalent to $(C, 0)$ it is necessary and sufficient that $p(z)$ should not have zeros on or within the unit circle.

II'. If $p(z)$ has zeros of maximum order $m$ on the unit circle and does not have zeros within the unit circle, then $(C, m)$ includes $N_{p}$, but, for any $\varepsilon>0$, there is an $N_{p}$-convergent sequence which is not $(C, m-\varepsilon)$-convergent.

III'. If $p(z)$ has a zero within the unit circle, then there is an $N_{p}$-convergent sequence which is not A-convergent.

Result $\mathrm{I}^{\prime}$ is essentially equivalent to a theorem due to Kubota (3).

Some of the principal results established by Boyd and myself in a recent paper (1) can be deduced from $\mathrm{II}^{\prime}$ by considering $p(z)=2^{-m}(1+z)^{m}$ and $p(z)=\alpha+\beta z+(1-\alpha-\beta) z^{2}$ with $\alpha, \beta$ real.

\section{Proof of Theorem III, and Lemmas}

Proof of Theorem III. We start with this theorem because its proof is simpler than those of Theorems I and II.

Since $1 / p(z)$ is analytic in a neighbourhood $U$ of the origin, there is a 
sequence $\left\{s_{n}\right\}$ such that, for $z$ in $U$,

Let

$$
\sum_{n=0}^{\infty} s_{n} z^{n}=1 / p(z)
$$

Then, for $z$ in $U$,

$$
t_{n}=\sum_{v=0}^{n} p_{v} s_{n-v}, \quad u_{n}=\sum_{v=0}^{n} q_{v} s_{n-v}
$$

and

$$
\sum_{n=0}^{\infty} t_{n} z^{n}=p(z) \sum_{n=0}^{\infty} s_{n} z^{n}=1
$$

$$
\sum_{n=0}^{\infty} u_{n} z^{n}=q(z) \sum_{n=0}^{\infty} s_{n} z^{n}=q(z) / p(z)
$$

Hence $t_{0}=1, t_{n}=0$ for $n>0$, and so $\left\{s_{n}\right\}$ is $N_{p}$-convergent to 0 . On the other hand $\Sigma u_{n} z^{n}$ has radius of convergence less than unity, because, by hypothesis, $q(z) / p(z)$ has a pole within the unit circle. Consequently, $\left\{u_{n}\right\}$ is not $A$-convergent and so $\left\{s_{n}\right\}$ is not $A N_{q}$-convergent.

We now prove two lemmas.

Lemma 1. If $q(z) / p(z)$ has poles $\lambda_{1}, \lambda_{2}, \ldots, \lambda_{l}$ (and no others) of orders $m_{1}, m_{2}, \ldots, m_{l}$, and if for $n=0,1, \ldots$,

then

$$
t_{n}=\sum_{v=0}^{n} p_{v} s_{n-v}, \quad u_{n}=\sum_{v=0}^{n} q_{v} s_{n-v}
$$

$$
u_{n}=\sum_{v=0}^{n} c_{v} t_{n-v}+\sum_{r=1}^{l} \sum_{\rho=1}^{m_{r}} c_{r, p} \sum_{v=0}^{n}\left(\begin{array}{c}
v+\rho-1 \\
\rho-1
\end{array}\right) \lambda_{r}^{-v} t_{n-v}
$$

where the c's are constants, depending only on $p_{0}, p_{1}, \ldots, p_{j}, q_{0}, q_{1}, \ldots, q_{k}$, such that $c_{n}=0$ for $n>k-j$ and $c_{r, m_{r}} \neq 0$.

Proof. Let $N$ be any positive integer, and let

$$
\begin{gathered}
s_{n}^{\prime}=\left\{\begin{array}{l}
s_{n} \text { for } 0 \leqq n \leqq N, \\
0 \text { for } n>N,
\end{array}\right. \\
t_{n}^{\prime}=\sum_{v=0}^{n} p_{v} s_{n-v}^{\prime}, \quad u_{n}^{\prime}=\sum_{v=0}^{n} q_{v} s_{n-v}^{\prime} ;
\end{gathered}
$$

so that $t_{n}^{\prime}=t_{n}, u_{n}^{\prime}=u_{n}$ for $0 \leqq n \leqq N$, and $t_{n}^{\prime}=u_{n}^{\prime}=0$ for $n>j+k+N$. Then

$$
\sum_{n=0}^{\infty} t_{n}^{\prime} z^{n}=p(z) \sum_{n=0}^{\infty} s_{n}^{\prime} z^{n}, \quad \sum_{n=0}^{\infty} u_{n}^{\prime} z^{n}=q(z) \sum_{n=0}^{\infty} s_{n}^{\prime} z^{n},
$$

and so, since 0 is not a pole of $q(z) / p(z)$,

$$
\begin{aligned}
\sum_{n=0}^{\infty} u_{n}^{\prime} z^{n} & =\frac{q(z)}{p(z)} \sum_{n=0}^{\infty} t_{n}^{\prime} z^{n} \\
& =\left\{\sum_{n=0}^{\infty} c_{n} z^{n}+\sum_{r=1}^{1} \sum_{\rho=1}^{m_{r}} c_{r, \rho}\left(1-\frac{z}{\lambda_{r}}\right)^{-\rho}\right\} \sum_{n=0}^{\infty} t_{n}^{\prime} z^{n}
\end{aligned}
$$


where $c_{n}=0$ for $n>k-j$ and $c_{r, m_{r}} \neq 0$. Expanding $\left(1-z / \lambda_{r}\right)^{-\rho}$, with $|z|<\min \left(\left|\lambda_{1}\right|,\left|\lambda_{2}\right|, \ldots,\left|\lambda_{l}\right|\right)$, and equating coefficients, we obtain the required identity for $0 \leqq n \leqq N$. Since $N$ can be taken arbitrarily large it must hold for all $n$.

Lemma 2. If $|\lambda|>1, \rho$ is any real number, and $t_{n} \rightarrow 0$, then

$$
\lim _{n \rightarrow \infty} \sum_{v=0}^{n}\left(\begin{array}{c}
v+\rho-1 \\
\rho-1
\end{array}\right) \lambda^{-v} t_{n-v}=0 .
$$

Since $\sum_{\nu=0}^{\infty}\left(\begin{array}{c}v+\rho-1 \\ \rho-1\end{array}\right) \lambda^{-v}$ is absolutely convergent, the result is evident.

\section{Proof of Theorem I, and Lemmas}

Proof of Theorem I (sufficiency). The hypothesis is that the function $q(z) / p(z)$ does not have poles on or within the unit circle. If it does not have any poles at all it must be a polynomial and so, by Theorem $2, N_{q}$ includes $N_{p}$. Otherwise, it follows from Lemmas 1 and 2 that $s_{n} \rightarrow 0\left(N_{q}\right)$ whenever $s_{n} \rightarrow 0\left(N_{p}\right)$, and hence that $s_{n} \rightarrow s\left(N_{q}\right)$ whenever $s_{n} \rightarrow s\left(N_{p}\right)$.

The necessity part of Theorem I is a consequence of Theorems II and III. It remains only to prove Theorem II and for this we require three additional lemmas.

Lemma 3. If $|\lambda|=1, \lambda \neq 1, \alpha>-1, \beta>-1$, then

$$
\begin{gathered}
\sum_{\nu=0}^{n}\left(\begin{array}{c}
n-v+\beta \\
\beta
\end{array}\right)\left(\begin{array}{c}
v+\alpha \\
\alpha
\end{array}\right) \lambda^{-v}=\left(\begin{array}{c}
n+\beta \\
\beta
\end{array}\right)(1-1 / \lambda)^{-\alpha-1}+\left(\begin{array}{c}
n+\alpha \\
\alpha
\end{array}\right) \lambda^{-n}(1-\lambda)^{-\beta-1} \\
+O\left(n^{\beta-1}+n^{\alpha-1}\right) .
\end{gathered}
$$

Here and elsewhere it is to be assumed that powers of complex numbers have their principal values.

A proof of the above lemma is given in (2), \$6.9. Using a similar method of proof we shall establish

Lemma 4. If $|\lambda|=|\mu|=1, \lambda \neq 1, \mu \neq 1, \lambda \neq \mu, \alpha>-1, \beta>-1$, $\gamma>-1$, and

then

$$
v_{n}=\sum_{\nu=0}^{n}\left(\begin{array}{c}
n-v+\beta \\
\beta
\end{array}\right) \mu^{v-n}\left(\begin{array}{c}
v+\alpha \\
\alpha
\end{array}\right) \lambda^{-v}
$$

$$
\begin{aligned}
w_{n}=\sum_{r=0}^{n}\left(\begin{array}{c}
r+\gamma \\
\gamma
\end{array}\right) v_{n-r} & =\left(\begin{array}{c}
n+\gamma \\
\gamma
\end{array}\right)(1-1 / \lambda)^{-\alpha-1}(1-1 / \mu)^{-\beta-1} \\
& +\left(\begin{array}{c}
n+\alpha \\
\alpha
\end{array}\right) \lambda^{-n}(1-\lambda)^{-\gamma-1}(1-\lambda / \mu)^{-\beta-1} \\
& +\left(\begin{array}{c}
n+\beta \\
\beta
\end{array}\right) \mu^{-n}(1-\mu)^{-\gamma-1}(1-\mu / \lambda)^{-\alpha-1} \\
& +O\left(n^{\gamma-1}+n^{\alpha-1}+n^{\beta-1}\right) .
\end{aligned}
$$


Proof. Note that, within the unit circle,

$$
\begin{aligned}
\sum_{n=0}^{\infty} w_{n} z^{n} & =(1-z / \lambda)^{-\alpha-1}(1-z / \mu)^{-\beta-1}(1-z)^{-\gamma-1} \\
& =w(z)
\end{aligned}
$$

say, so that

$$
2 \pi i w_{n}=\int_{C} w(z) z^{-n-1} d z
$$

where $C$ is the circle $|z|=\rho<1$. Let $z_{1}=1, z_{2}=\lambda, z_{3}=\mu$, and let $n>1 / \delta$ where $\delta=\min \left(\left|z_{1}-z_{2}\right|,\left|z_{2}-z_{3}\right|,\left|z_{3}-z_{1}\right|\right)$. Then, by Cauchy's theorem,

$$
2 \pi i w_{n}=\sum_{r=1}^{3} \int_{C_{r}} w(z) z^{-n-1} d z
$$

where $C_{r}$ is the contour formed by the circle $\left|z-z_{r}\right|=1 / n$ and the infinite segment $z=z_{r} \tau, \tau \geqq 1+1 / n$, the latter being described twice.

Let

$$
u(z)=(1-1 / \lambda)^{-\alpha-1}(1-1 / \mu)^{-\beta-1}(1-z)^{-\gamma-1} ;
$$

so that

$$
\begin{aligned}
\int_{C_{1}} u(z) z^{-n-1} d z & =\int_{C} u(z) z^{-n-1} d z \\
& =2 \pi i(1-1 / \lambda)^{-\alpha-1}(1-1 / \mu)^{-\beta-1}\left(\begin{array}{c}
n+\gamma \\
\gamma
\end{array}\right) .
\end{aligned}
$$

Further, for $z$ on $C_{1}$,

$$
\begin{aligned}
w(z)-u(z) & =(1-z)^{-\gamma-1} \int_{1}^{z}\left\{\lambda^{-1}(\alpha+1)(1-t / \lambda)^{-\alpha-2}(1-t / \mu)^{-\beta-1}\right. \\
& =O\left(|z-1|^{-\gamma}\right) .
\end{aligned}
$$

Consequently, the contribution of the circle to

$$
\int_{C_{1}}\{w(z)-u(z)\} z^{-n-1} d z
$$

is $O\left\{(1 / n)^{-\gamma}(1 / n)\right\}=O\left(n^{\gamma-1}\right)$, and that of the rest of $C_{1}$ (see (2), 138) is

$$
O\left\{\int_{1+1 / n}^{\infty}(x-1)^{-\gamma} x^{-n-1} d x\right\}=O\left(n^{\gamma-1}\right)
$$

Hence

$$
\begin{aligned}
\int_{C_{1}} w(z) z^{-n-1} d z & =\int_{C_{1}}(1-z / \lambda)^{-\alpha-1}(1-z / \mu)^{-\beta-1}(1-z)^{-\gamma-1} z^{-n-1} d z \\
& =2 \pi i(1-1 / \lambda)^{-\alpha-1}(1-1 / \mu)^{-\beta-1}\left(\begin{array}{c}
n+\gamma \\
\gamma
\end{array}\right)+O\left(n^{\gamma-1}\right)
\end{aligned}
$$


Now

$$
\begin{aligned}
\int_{C_{2}} w(z) z^{-n-1} d z & =\int_{C_{1}} w(\lambda z)(\lambda z)^{-n-1} \lambda d z \\
& =\lambda^{-n} \int_{C_{1}}(1-z)^{-\alpha-1}(1-\lambda z / \mu)^{-\beta-1}(1-\lambda z)^{-\gamma-1} z^{-n-1} d z \\
& =2 \pi i \lambda^{-n}(1-\lambda)^{-\gamma-1}(1-\lambda / \mu)^{-\beta-1}\left(\begin{array}{c}
n+\alpha \\
\alpha
\end{array}\right)+O\left(n^{\alpha-1}\right)
\end{aligned}
$$

by (2), since $|\mu / \lambda|=1, \mu / \lambda \neq 1,1 / \lambda \neq 1, \mu / \lambda \neq 1 / \lambda$.

Similarly,

$$
\int_{C_{3}} w(z) z^{-n-1} d z=2 \pi i \mu^{-n}(1-\mu)^{-\gamma-1}(1-\mu / \lambda)^{-\alpha-1}\left(\begin{array}{c}
n+\beta \\
\beta
\end{array}\right)+O\left(n^{\beta-1}\right) .
$$

The required conclusion follows from the numbered identities.

Lemma 5. If $|\lambda|=1, \lambda \neq 1, \alpha>-1$ and $t_{n} \rightarrow 0$, then

$$
v_{n}=\sum_{v=0}^{n}\left(\begin{array}{c}
v+\alpha \\
\alpha
\end{array}\right) \lambda^{-v} t_{n-v} \rightarrow 0(C, \alpha+1) \text {. }
$$

Proof. We have

$$
\sum_{r=0}^{n}\left(\begin{array}{c}
r+\alpha \\
\alpha
\end{array}\right) v_{n-r}=\sum_{r=0}^{n} t_{n-r} \sum_{v=0}^{r}\left(\begin{array}{c}
r-v+\alpha \\
\alpha
\end{array}\right)\left(\begin{array}{c}
v+\alpha \\
\alpha
\end{array}\right) \lambda^{-v}
$$

which, by Lemma 3 , is

$$
o\left\{\sum_{r=0}^{n}\left|t_{n-r}\right|\left(\begin{array}{c}
r+\alpha \\
\alpha
\end{array}\right)\right\}=o\left\{\left(\begin{array}{c}
n+\alpha+1 \\
\alpha+1
\end{array}\right)\right\}
$$

and this is the required conclusion.

\section{Proof of Theorem II}

Let

$$
t_{n}=\sum_{v=0}^{n} p_{v} s_{n-v}, \quad u_{n}=\sum_{v=0}^{n} q_{v} s_{n-v} .
$$

Our hypothesis is that the function $q(z) / p(z)$ has poles of maximum order $m$ on the unit circle and that its other poles (if any) lie outside the unit circle. Also $p(1)=1$ and so $z=1$ is not a pole of $q(z) / p(z)$. Hence, by Lemmas 1,2 and 5, if $s_{n} \rightarrow 0\left(N_{p}\right)$, i.e. if $t_{n} \rightarrow 0$, then $u_{n} \rightarrow 0(C, m)$, i.e. $s_{n} \rightarrow 0(C, m) N_{q}$. Since all the summability methods concerned are regular, it follows that $s_{n} \rightarrow s$ $(C, m) N_{q}$ whenever $s_{n} \rightarrow s\left(N_{p}\right)$, i.e. that $(C, m) N_{q}$ includes $N_{p}$.

We have thus established the first part of Theorem II. To prove the remainder, suppose, as we may without loss in generality, that

$$
0<\varepsilon<1 \text {. }
$$

Let the poles of $q(z) / p(z)$ be $\lambda_{1}, \lambda_{2}, \ldots, \lambda_{l}$ with orders $m_{1}, m_{2}, \ldots, m_{l}$. Suppose 
the numbering to be such that the first $l^{\prime}$ of these are the ones on the unit circle and that

$$
m_{1}=m=\max \left(m_{1}, m_{2}, \ldots, m_{l^{\prime}}\right) .
$$

Let $\left\{s_{n}\right\}$ be the sequence for which

$$
t_{n}=\lambda_{1}^{-n}\left(\begin{array}{c}
n-\varepsilon \\
-\varepsilon
\end{array}\right)
$$

the existence (and uniqueness) of the sequence $\left\{s_{n}\right\}$ being ensured by the condition $p_{0}=p(0) \neq 0$. Then, taking $c_{r, \rho}$ to be 0 if $\rho>m_{r}$, we have, by Lemma 1 ,

$$
u_{n}=\sum_{\tau=1}^{4} u_{n}^{(\tau)}
$$

where

$$
\begin{aligned}
& u_{n}^{(1)}=\left\{\begin{array}{l}
\sum_{v=0}^{n} c_{v} t_{n-\nu}+\sum_{r=l^{\prime}+1}^{l} \sum_{\rho=1}^{m_{r}} c_{r, \rho} \sum_{\nu=0}^{n}\left(\begin{array}{c}
\nu+\rho-1 \\
\rho-1
\end{array}\right) \lambda_{r}^{-v} t_{n-v} \text { if } l>l^{\prime}, \\
\sum_{v=0}^{n} c_{v} t_{n-v} \text { if } l=l^{\prime} ;
\end{array}\right. \\
& u_{n}^{(2)}=\left\{\begin{array}{l}
\sum_{r=1}^{l^{\prime}} \sum_{\rho=1}^{m-1} c_{r, \rho} \sum_{v=0}^{n}\left(\begin{array}{c}
v+\rho-1 \\
\rho-1
\end{array}\right) \lambda_{r}^{-v} t_{n-v} \text { if } m>1, \\
0 \text { if } m=1 ;
\end{array}\right. \\
& u_{n}^{(3)}=\left\{\begin{array}{l}
\sum_{r=2}^{l^{\prime}} c_{r, m} \sum_{\nu=0}^{n}\left(\begin{array}{c}
v+m-1 \\
m-1
\end{array}\right) \lambda_{r}^{-v}\left(\begin{array}{c}
n-v-\varepsilon \\
-\varepsilon
\end{array}\right) \lambda_{1}^{v-n} \text { if } l^{\prime}>1, \\
0 \text { if } l^{\prime}=1 ;
\end{array}\right. \\
& u_{n}^{(4)}=c_{1, m} \lambda_{1}^{-n} \sum_{\nu=0}^{n}\left(\begin{array}{c}
v+m-1 \\
m-1
\end{array}\right)\left(\begin{array}{c}
n-v-\varepsilon \\
-\varepsilon
\end{array}\right)=c_{1, m} \lambda_{1}^{-n}\left(\begin{array}{c}
n+m-\varepsilon \\
m-\varepsilon
\end{array}\right) \text {. }
\end{aligned}
$$

Now $t_{n} \rightarrow 0, c_{v}=0$ for $v>k-j$, and $\left|\lambda_{r}\right|>1$ if $l \geqq r>l^{\prime}$ : hence, by Lemma 2 ,

$$
u_{n}^{(1)} \rightarrow 0 \text {. }
$$

Further, $\left|\lambda_{r}\right|=1, \lambda_{r} \neq 1$ for $r=1,2, \ldots, l^{\prime}$, so that, by Lemma 5 ,

$$
u_{n}^{(2)} \rightarrow 0(C, m-1) \text {; }
$$

and, by Lemma 4 ,

$$
u_{n}^{(3)} \rightarrow 0(C, m-\varepsilon),
$$

since $m-\varepsilon>\max (m-1,-\varepsilon)$ and $m-\varepsilon-1>-1$.

Consequently $u_{n}-u_{n}^{(4)} \rightarrow 0(C, m-\varepsilon)$; but, by Lemma 3 (or by Theorem 46 in (2), since $\left.u_{n}^{(4)} \neq o\left(n^{m-\varepsilon}\right)\right), u_{n}^{(4)}$ does not tend to a limit $(C, m-\varepsilon)$. The sequence $\left\{u_{n}\right\}$ is therefore not $(C, m-\varepsilon)$-convergent; so that the sequence $\left\{s_{n}\right\}$ is not $(C, m-\varepsilon) N_{q}$-convergent though it is $N_{p}$-convergent to 0 . 


\section{REFERENCES}

(1) D. BoRWErn and A. V. BOYD, Binary and ternary transformations of sequences, Proc. Edin. Math. Soc., 11 (1959), 175-181.

(2) G. H. Hardy, Divergent Series (Oxford, 1949).

(3) T. КuвотA, Ein Satz über den Grenzwert, Tôhoku Math. Journal, 12 (1917), 222-224.

(4) L. L. Silverman and O. Szasz, On a class of Nörlund matrices, Annals of Math., 45 (1944), 347-357.

St Salvator's College

UNIVERSITY OF ST ANDREWS 